\title{
Lung Adenocarcinoma, Mixed Subtype
}

National Cancer Institute

\section{Source}

National Cancer Institute. Lung Adenocarcinoma, Mixed Subtype. NCI Thesaurus. Code C45508.

The most frequently seen morphologic variant of lung adenocarcinoma characterized by a mixture of architectural patterns, including acinar, papillary, bronchioloalveolar, and solid pattern. 